OU-HET 184

December 1993

\title{
Wheeler-DeWitt Equation in Two-dimensional Quantum Gravity with $c=1$ Conformal Matter Field
}

\author{
Atushi Ishikawa円 \\ Department of Physics \\ Osaka University, Toyonaka, Osaka 560, Japan
}

\begin{abstract}
In the context of two-dimensional quantum cosmology, we consider the pathintegral of a string on annulus which contains the Liouville field and conformal matter fields. We show that, in the transition amplitude of the string universe, the non-zero modes of the fields are all cancelled out only when we take the $c=1$ conformal matter field and impose the Neumann boundary condition on the system. The transition amplitude obtained obeys the minisuperspace Wheeler-DeWitt equation. In our treatment, the modular parameter on annulus plays the role of time variable to integrate out.
\end{abstract}

\footnotetext{
${ }^{1}$ e-mail: ishikawa@oskth.kek.jp
} 


\section{Introduction}

It is well-known that string theory can be viewed as two-dimensional gravity. Here we want to consider it as a toy model for four-dimensional quantum cosmology.

In four dimensions, a quantum state of universe is described by a wave function of universe on superspace (see for example Ref.[1]). Superspace has a finite number of coordinates at every point on the three-hypersurface and is infinite-dimensional. In order to discuss quantum mechanical properties of universe, many people want to solve zeroenergy Schrödinger equations that are decomposed into the Wheeler-DeWitt equation and the momentum constraints by the $(3+1)$ decomposition consisting of the lapse and shift functions $\left(N, N^{i}\right)$ and the three-metric on a hypersurface $\left(h_{i j}\right)$. It is very hard, however, to solve such infinite-dimensional differential equations without any approximation apart from many difficult conceptual problems. In order to make this problem tractable, we often reduce the degrees of freedom of the superspace to a finite number by assuming a symmetry. This reduced finite dimensional superspace is called minisuperspace. In the minisuperspace, the transition amplitude of universe defined by the path-integral can be easily shown to obey the Wheeler-DeWitt equation in the $\dot{N}=0$ gauge [0].

In contrast to the above four-dimensional case, the Einstein action becomes a topological number in two dimensions. In order to obtain a gravitational theory, we have to treat an anomaly of the path-integral measure exactly (see for example Ref.[3]). It has been proposed that the minisuperspace represents the superspace exactly in two dimensions $\mathbb{\|}$, [5]. The reason may be that an argument of a wave function of one-dimensional universe may be the length of the universe itself. This conjecture is partially supported by the calculations of the $c=0$ matrix model $[4]$.

In this paper, in the context of quantum cosmology, we want to investigate this proposal in the framework of the continuum Liouville theory. In $\S 2$, in order to investigate the lapse, shift and the Liouville field in two-dimensional gravity generally, we start from the Polyakov action and briefly review the known formulation to rewrite it by using the $(1+1)$ decomposition [6]. Some of this discussion in what follows is developed in Ref. [7].

We show that the path-integral measure $\left[\mathcal{D} g_{a b}\right]$ can be decomposed into $[\mathcal{D} N][\mathcal{D} M][\mathcal{D} \phi]$ by using the lapse and shift functions $(N, M)$ and the Liouville field $(\phi)$. There is an 
undesirable term consisting only of $N$ and $M$ in the Liouville action however.

Next, in $\S 3$, we take a conformal gauge in order to eliminate the problematic term above. We restrict ourselves to the case of annulus topology and calculate the transition amplitude of the one-dimensional loop universe. We show that the non-zero modes of the fields are all cancelled out in the transition amplitude only when we take the $c=1$ conformal matter field and impose the Neumann boundary condition on the system. The reason is as follows. When the cosmological constant is ignored, the Liouville field acts as an extra conformal matter field. Therefore a string on the worldsheet cannot vibrate in the two-dimensional target space. The rational for ignoring the cosmological constant will be discussed later in this section. This cancellation of the non-zero modes is similar to the case of torus topology which was investigated by Bershadsky and Klebanov [8]. Our guiding principle is that ghost fields should not appear on the boundary in the context of quantum cosmology, and this requirement is satisfied only by the Neumann boundary condition. In open string theory, the Neumann boundary condition is taken, because the end points of a string are free. In our case, the Neumann boundary condition means that we must sum over all allowed values of the non-zero modes in the initial and final states. As a result of these settings, we obtain the transition amplitude constructed only by the zero modes. It can be said that the Wheeler-DeWitt equation, that this transition amplitude obeys, exists only on the minisuperspace, and therefore the minisuperspace represents the superspace exactly. Our result crucially depends on the fact that we consider the case with the $c=1$ conformal matter field.

\section{The Lapse, Shift and the Liouville Field in Two- dimensional Gravity}

In this section, we will briefly review the formulation of two-dimensional gravity (see for example Ref.[3]). Starting from the Polyakov action, we will rewrite it by the $(1+1)$ decomposition. We will obtain the transition amplitude of the sting universe, which will be used in $\S 3$.

We write the coordinates on the worldsheet as $\xi^{a}=\left(\xi^{0}, \xi^{1}\right)$. In this coordinate, the 
transition amplitude is represented as

$$
Z\left[X_{F}^{\mu}, \phi_{F} ; X_{I}^{\mu}, \phi_{I}\right]=\int \frac{\left[\mathcal{D} g_{a b}\right]\left[\mathcal{D} X^{\mu}\right]}{\operatorname{vol}(\text { Gauge })} \exp \left\{-\frac{1}{2} \int d^{2} \xi \sqrt{g} g^{a b} \partial_{a} X^{\mu} \partial_{b} X_{\mu}\right\},
$$

where the metric on the worldsheet is given by

$$
d s^{2}=g_{a b}(\xi) d \xi^{a} d \xi^{b}=e^{\phi(\xi)} \hat{g}_{a b}(\xi) d \xi^{a} d \xi^{b}
$$

Here we take a conformal time on the worldsheet, and can take out the dynamical variable as an overall conformal mode. We also parametrize the fiducial metric $\hat{g}_{a b}(\xi)$ by using the lapse and shift functions $N(\xi), M(\xi)$, following the ADM decomposition in two dimensions [6];

$$
\hat{g}_{a b}(\xi)=\left(\begin{array}{cc}
N(\xi)^{-2}+M(\xi)^{2} & M(\xi) \\
M(\xi) & 1
\end{array}\right) .
$$

The reason why we take an inverse of the lapse function will be discussed later. The most general local metric on deformations $\delta g_{a b}$ of the metric is given by $\|\delta g\|^{2}=\int d^{2} \xi \sqrt{g}\left\{G^{a b c d}+\right.$ $\left.u g^{a b} g^{c d}\right\} \delta g_{a b} \delta g_{c d}$, where $u$ is an arbitrary positive real number and $G^{a b c d}$ is the identity operator in the space of symmetric traceless tensors. The decomposition of the measure $\left[\mathcal{D} g_{a b}\right]$ in the transition amplitude (2.1) is given by the orthogonal decomposition; $\delta g_{a b}=\delta h_{a b}+(\delta \rho) g_{a b}$, where

$$
\delta \rho=-\frac{\delta N}{N}+\delta \phi
$$

and

$$
\delta h_{a b}=e^{\phi}\left(\begin{array}{cc}
\left(\frac{M^{2}}{N}-\frac{1}{N^{3}}\right) \delta N+2 M \delta M & \frac{M}{N} \delta N+\delta M \\
\frac{M}{N} \delta N+\delta M & \frac{\delta N}{N}
\end{array}\right) .
$$

Here $\delta \rho$ is the trace part of the metric deformations $\delta g_{a b}$, and $\delta h_{a b}$ is the symmetric traceless part. Then the metric on deformations of $\delta g_{a b}$ is decomposed as

$$
\|\delta g\|^{2}=\int d^{2} \xi \sqrt{g} G^{a b c d} \delta h_{a b} \delta h_{c d}+4 u \int d^{2} \xi \sqrt{g}(\delta \rho)^{2} .
$$

From this decomposition, we can separate the measure $\left[\mathcal{D} g_{a b}\right]$ in the form of $[\mathcal{D} \rho]\left[\mathcal{D} h_{a b}\right]$. Next we change variables from $\rho, h_{a b}$ to $\phi, v_{a} ; \delta \rho=\delta \phi+g^{a b} \nabla_{a}\left(\delta v_{b}\right), \delta h_{a b}=2 G_{a b}^{c d} \nabla_{c}$ $\left(\delta v_{d}\right)=\left(P_{1} \delta v\right)_{a b}$, where $\delta v_{a}$ are infinitesimal generators with respect to a two-dimensional 
diffeomorphism. The operator $P_{1}$ maps vectors into symmetric traceless tensors. We obtain another decomposition with the Jacobian $\left\{\operatorname{det}^{\prime}\left(P_{1}^{\dagger} P_{1}\right)\right\}_{g}^{1 / 2}$;

$$
\left[\mathcal{D} g_{a b}\right]=\prod_{i} d \tau_{i} \frac{1}{\operatorname{vol}(\mathrm{CK})} \frac{<\psi^{(j)} \mid \frac{\partial g}{\partial \tau_{k}}>_{g}}{<\psi^{(j)} \mid \psi^{(k)}>_{g}^{1 / 2}}[\mathcal{D} \phi]\left[\mathcal{D} v_{a}\right]\left\{\operatorname{det}^{\prime}\left(P_{1}^{\dagger} P_{1}\right)\right\}_{g}^{1 / 2}
$$

where $\tau_{i}$ are moduli parameters and the factor $\frac{\left\langle\psi^{(j)} \mid \frac{\partial g}{\partial \tau_{k}}\right\rangle_{g}}{\left\langle\psi^{(j)} \mid \psi^{(k)}\right\rangle_{g}^{1 / 2}}$ is the Weil-Petersson measure which represents the angle between the moduli space and the gauge orbit of a diffeomorphism. The prime in equation (2.7) denotes the omission of zero-mode with respect to conformal Killing vectors $d V ; P_{1}(d V)=0$. We must divide by the volume of conformal Killing vectors $\operatorname{vol}(\mathrm{CK})$. The Liouville action $S_{\phi}$ arises in the formula;

$$
\frac{<\psi^{(j)} \mid \frac{\partial g}{\partial \tau_{k}}>_{g}}{<\psi^{(j)} \mid \psi^{(k)}>_{g}^{1 / 2}}\left\{\operatorname{det}^{\prime}\left(P_{1}^{\dagger} P_{1}\right)\right\}_{g}^{1 / 2}=\frac{<\psi^{(j)} \mid \frac{\partial \hat{g}}{\partial \tau_{k}}>_{\hat{g}}}{<\psi^{(j)} \mid \psi^{(k)}>_{\hat{g}}^{1 / 2}}\left\{\operatorname{det}^{\prime}\left(P_{1}^{\dagger} P_{1}\right)\right\}_{\hat{g}}^{1 / 2} e^{-\frac{26}{48 \pi} S_{\phi}[\phi, \hat{g}]} .
$$

Accordingly the measure is decomposed as

$$
\begin{aligned}
{\left[\mathcal{D} g_{a b}\right] } & =\prod_{i} d \tau_{i} \frac{<\psi^{(j)} \mid \frac{\partial \hat{g}}{\partial \tau_{k}}>_{\hat{g}}}{<\psi^{(j)} \mid \psi^{(k)}>_{\hat{g}}^{1 / 2}}[\mathcal{D} \phi]\left[\mathcal{D} v_{a}\right]\left\{\operatorname{det}^{\prime}\left(P_{1}^{\dagger} P_{1}\right)\right\}_{\hat{g}}^{1 / 2} e^{-\frac{26}{48 \pi} S_{\phi}[\phi, \hat{g}]} \\
& \equiv[\mathcal{D} \phi]\left[\mathcal{D} \hat{h}_{a b}\right] e^{-\frac{26}{48 \pi} S_{\phi}[\phi, \hat{g}]}
\end{aligned}
$$

where $\hat{h}_{a b}$ is defined by above equation. The most general local metric on deformations $\delta \hat{h}_{a b}$ is given by

$$
\begin{aligned}
\|\delta \hat{h}\|^{2} & =\int d^{2} \xi \sqrt{\hat{g}}\left\{\hat{G}^{a b c d}+u \hat{g}^{a b} \hat{g}^{c d}\right\} \delta \hat{h}_{a b} \delta \hat{h}_{c d} \\
& =\int d^{2} \xi\left\{2 \frac{(\delta N)^{2}}{N^{2}}+2 N^{2}(\delta M)^{2}\right\} .
\end{aligned}
$$

From this decomposition, we obtain another separation of the measure $\left[\mathcal{D} g_{a b}\right]$;

$$
\left[\mathcal{D} g_{a b}\right]=[\mathcal{D} \phi][\mathcal{D} N][\mathcal{D} M] e^{-\frac{26}{48 \pi} S_{\phi}[\phi, \hat{g}]}
$$

In obtaining this form, we have taken the parametrization of the fiducial metric given by equation (2.3). Here we find the same separation form of the measure that we take in four dimensions. This discussion is developed in Ref. [7]. We should be careful about treating the measure of the Liouville field $[\mathcal{D} \phi]$, because it is not translationally invariant. We will discuss it in $\S 3$. 
The difference of the path-integral for conformal matter fields (central charge $c$ ) evaluated on $g_{a b}$ and that on $\hat{g}_{a b}$ is also represented in terms of the Liouville action. Consequently the transition amplitude (2.1) can be expressed as

$$
\begin{aligned}
Z\left[X_{F}^{\mu}, \phi_{F} ; X_{I}^{\mu}, \phi_{I}\right]= & \int \frac{[\mathcal{D} N][\mathcal{D} M]}{\operatorname{vol}(\text { Gauge })} \int\left[\mathcal{D} X^{\mu}\right] \exp \left\{-\frac{1}{2} \int d^{2} \xi \sqrt{\hat{g}} \hat{g}^{a b} \partial_{a} X^{\mu} \partial_{b} X_{\mu}\right\} \\
& \times \int[\mathcal{D} \phi] e^{-\frac{26-c}{48 \pi} S_{\phi}[\phi, \hat{g}]} .
\end{aligned}
$$

By using the parametrization of the fiducial metric (2.3), we can write the action of conformal matter fields as

$$
\begin{aligned}
S_{X^{\mu}} & =\frac{1}{2} \int d^{2} \xi \sqrt{\hat{g}} \hat{g}^{a b} \partial_{a} X^{\mu} \partial_{b} X_{\mu} \\
& =\int d^{2} \xi\left\{P_{X_{\mu}} \dot{X}^{\mu}-N^{-1} H_{0}^{X^{\mu}}-M H_{1}^{X^{\mu}}\right\},
\end{aligned}
$$

where

$$
\begin{aligned}
P_{X}{ }^{\mu} & =N\left(\dot{X}^{\mu}-M X^{\mu \prime}\right), \\
H_{0}^{X^{\mu}} & =\frac{1}{2}\left(P_{X}{ }^{\mu} P_{X \mu}-X^{\mu^{\prime}} X_{\mu}{ }^{\prime}\right), \quad H_{1}^{X^{\mu}}=P_{X \mu} X^{\mu \prime} .
\end{aligned}
$$

Likewise,

$$
\begin{aligned}
S_{\phi} & =\kappa \int d^{2} \xi \sqrt{\hat{g}}\left\{\frac{1}{2} \hat{g}^{a b} \partial_{a} \phi \partial_{b} \phi+\hat{R} \phi+\mu e^{\phi}\right\} \\
& =\int d^{2} \xi\left\{P_{\phi} \dot{\phi}-N^{-1} H_{0}^{\phi}-M H_{1}^{\phi}+2 \kappa N M^{\prime 2}\right\}
\end{aligned}
$$

where

$$
\begin{aligned}
P_{\phi} & =\kappa N\left(\dot{\phi}-M \phi^{\prime}-2 M^{\prime}\right), \\
H_{0}^{\phi} & =\frac{1}{2 \kappa} P_{\phi}^{2}-\frac{\kappa}{2} \phi^{\prime 2}+2 \kappa \phi^{\prime \prime}+\mu e^{\phi}, \quad H_{1}^{\phi}=P_{\phi} \phi^{\prime}-2 \phi^{\prime} .
\end{aligned}
$$

The dot and the prime represent the derivative with respect to $\xi^{0}$ and $\xi^{1}$ respectively, and we take $\kappa=\frac{26-c}{48 \pi}$.

The extra term $2 \kappa N M^{\prime 2}$ in the Liouville action (2.15) prevents us from interpreting the lapse and shift functions as the Lagrange multipliers [6], [7], [9]. This is related to the fact that there is no invariance under the diffeomorphisms of the fiducial metric $\hat{g}_{a b}$ 
where the Liouville mode $\phi$ is taken out. Therefore we cannot construct the canonical formulation of this system; the degrees of freedom are insufficient and there is no canonical structure (classically). In order to avoid this problem, Teitelboim added an extra field to the system in order to recover the canonical structure [6], [9], [10]. The meaning of this extra field is unclear however. In the next section, we will bypass this problem for the case of annulus topology.

\section{The Transition Amplitude for the case of annulus}

In this section, we concentrate on the case of annulus topology and fix the diffeomorphisms completely by taking the easiest gauge, namely the conformal gauge, in order to eliminate the problematic term mentioned in the previous section. This gauge fixing is quite different from the $\dot{N}=0$ gauge used in the minisuperspace treatment of a four-dimensional gauge fixing (see for example Ref. [1] [2]). If we fix the diffeomorphisms completely, this problematic term disappears and the degrees of freedom in the fiducial metric $\hat{g}_{a b}$ become finite, and is described by a modular parameter $t$ in the annulus case. In other words, we can take $N^{-1}=t$ and $M=0$ in (2.3). In this gauge, from the decomposition of the measure $\left[\mathcal{D} g_{a b}\right]$ given by the first line of equation (2.9), we obtain the transition amplitude of the one-dimensional loop universe;

$$
\begin{aligned}
Z\left[X_{F}^{\mu}, \phi_{F} ; X_{I}^{\mu}, \phi_{I}\right]= & \int_{0}^{\infty} d t \frac{1}{\Omega(C K)} \frac{<\psi \mid \frac{\partial \hat{g}}{\partial t}>_{\hat{g}}}{<\psi \mid \psi>_{\hat{g}}^{1 / 2}}\left\{\operatorname{det}^{\prime}\left(P_{1}^{\dagger} P_{1}\right)\right\}_{\hat{g}}^{1 / 2} \\
& \times \int\left[\mathcal{D} X^{\mu}\right] e^{-S_{X^{\mu}}\left[X^{\mu}, t\right]} \int[\mathcal{D} \phi] e^{-S_{\phi}[\phi, t]} \\
= & \int_{0}^{\infty} \frac{d t}{t}\left\{\operatorname{det}^{\prime}\left(P_{1}^{\dagger} P_{1}\right)\right\}_{\hat{g}}^{1 / 2} \int\left[\mathcal{D} X^{\mu}\right] e^{-S_{X^{\mu}\left[X^{\mu}, t\right]} \int[\mathcal{D} \phi] e^{-S_{\phi}[\phi, t]}}
\end{aligned}
$$

where we have used the following results of the calculations of the Weil-Petersson measure and the volume of a conformal Killing vector;

$$
\frac{\left\langle\psi \mid \frac{\partial \hat{g}}{\partial t}\right\rangle_{\hat{g}}}{\langle\psi| \psi>_{\hat{g}}^{1 / 2}}=\left(\frac{2}{t}\right)^{1 / 2}
$$

and

$$
\Omega(C K)=t^{1 / 2} .
$$


Note that the volume of $v_{a}$ is divided by $\operatorname{vol}$ (Gauge). Here the action of the conformal matter fields and that of the Liouville field can be rewritten respectively as

$$
S_{X^{\mu}}\left[X^{\mu}, t\right]=\frac{1}{2} \int_{M} d^{2} \sigma\left\{\dot{X}^{\mu} \dot{X}_{\mu}+X^{\mu \prime} X_{\mu}^{\prime}\right\}
$$

and

$$
S_{\phi}[\phi, t]=\frac{\kappa}{2} \int_{M} d^{2} \sigma\left\{\dot{\phi}^{2}+\phi^{\prime 2}-4 \phi^{\prime \prime}\right\}
$$

Here we have simply discarded the cosmological constant (see the discussion later for the case of $c=1$ ).

In order to obtain the actions (3.4) and (3.5), we have made a coordinate transformation from $\xi^{a}$ to $\sigma^{a}\left(\sigma^{0}=t \xi^{0}, \sigma^{1}=\xi^{1}\right)$ and the region $M$ of the new coordinates $\sigma^{a}$ is given by

$$
M: \quad 0 \leq \sigma^{0} \leq t, \quad 0 \leq \sigma^{1} \leq 1
$$

Here the space coordinate $\sigma^{1}$ is periodic and the boundaries exist at $\sigma^{0}=0, t$. From this transformation, we can interpret the modular parameter $t$ as a time variable of the system consisting of conformal matter fields and the Liouville field.

For the computation of the transition amplitude (3.1), we will make the mode expansion. For conformal matter fields, we expand them as follows

$$
X^{\mu}\left(\sigma^{0}, \sigma^{1}\right)=X_{0}^{\mu}\left(\sigma^{0}\right)+\sum_{n \neq 0} a_{n}^{\mu}\left(\sigma^{0}\right) e^{-2 \pi i \sigma^{1}}
$$

The first term is the zero mode and the second term represents the vibrations. We can separate the partition function of matter fields into the zero mode part and the non-zero modes parts;

$$
\begin{aligned}
\int\left[\mathcal{D} X^{\mu}\right] e^{-S_{X^{\mu}}\left[X^{\mu}, t\right]} & =\int\left[\mathcal{D} X_{0}^{\mu}\right] e^{-S_{X_{0}^{\mu}}^{\mu}\left[X_{0}^{\mu}, t\right]} \prod_{n \neq 0} \int\left[\mathcal{D} a_{n}^{\mu}\right] e^{-S_{a_{n}^{\mu}}\left[a_{n}^{\mu}, t\right]} \\
& =K\left(X_{0 F}^{\mu}, t ; X_{0 I}^{\mu}, 0\right) \prod_{n \neq 0}\left\{\frac{1}{n \sinh (2 \pi n t)}\right\}^{c / 2}
\end{aligned}
$$


where

$$
\begin{aligned}
S_{X^{\mu}}\left[X^{\mu}, t\right] & =S_{X_{0}^{\mu}}\left[X_{0}^{\mu}, t\right]+\sum_{n \neq 0} S_{a_{n}^{\mu}}\left[a_{n}^{\mu}, t\right] \\
S_{X_{0}^{\mu}}\left[X_{0}^{\mu}, t\right] & =\frac{1}{2} \int_{0}^{t} d \sigma^{0} \dot{X}_{0}^{\mu} \dot{X}_{0 \mu} \\
S_{a_{n}^{\mu}}\left[a_{n}^{\mu}, t\right] & =\frac{1}{2} \int_{0}^{t} d \sigma^{0}\left\{\dot{a}_{n}^{\mu} \dot{a}_{n \mu}+(2 \pi n)^{2} a_{n}^{\mu} a_{n \mu}\right\}
\end{aligned}
$$

and

$$
\begin{aligned}
K\left(X_{0 F}^{\mu}, t ; X_{0 I}^{\mu}, 0\right) & \equiv \int\left[\mathcal{D} X_{0}^{\mu}\right] e^{-S_{X_{0}^{\mu}}\left[X_{0}^{\mu}, t\right]} \\
& =\left(\frac{1}{2 \pi t}\right)^{c / 2} \exp \left\{-\frac{\left(X_{0 F}^{\mu}-X_{0 I}^{\mu}\right)\left(X_{0 F \mu}-X_{0 I \mu}\right)}{2 t}\right\}, \\
\int\left[\mathcal{D} a_{n}^{\mu}\right] e^{-S_{a_{n}^{\mu}}\left[a_{n}^{\mu}, t\right]} & =\left\{\frac{1}{n \sinh (2 \pi n t)}\right\}^{c / 2} .
\end{aligned}
$$

We use the knowledge of the path-integral in quantum mechanics for a free particle and a harmonic oscillator. Here we take the Neumann boundary condition as stated in the introduction. Note that the Neumann boundary condition puts no restriction on the zero mode. For the Liouville field, we also make the same mode expansion;

$$
\phi\left(\sigma^{0}, \sigma^{1}\right)=\phi_{0}\left(\sigma^{0}\right)+\sum_{n \neq 0} b_{n}\left(\sigma^{0}\right) e^{-2 \pi i \sigma^{1}}
$$

We also separate the partition function of the Liouville field into two parts;

$$
\int[\mathcal{D} \phi] e^{-S_{\phi}[\phi, t]}=K\left(\phi_{0 F}, t ; \phi_{0 I}, 0\right) \prod_{n \neq 0}\left\{\frac{1}{n \sinh (2 \pi n t)}\right\}^{1 / 2},
$$

where

$$
\begin{aligned}
S_{\phi}[\phi, t] & =S_{\phi_{0}}\left[\phi_{0}, t\right]+\sum_{n \neq 0} S_{b_{n}}\left[b_{n}, t\right], \\
S_{\phi_{0}}\left[\phi_{0}, t\right] & =\frac{\kappa}{2} \int_{0}^{t} d \sigma^{0} \dot{\phi}_{0}^{2}, \\
S_{b_{n}}\left[b_{n}, t\right] & =\frac{\kappa}{2} \int_{0}^{t} d \sigma^{0}\left\{{\dot{b_{n}}}^{2}+(2 \pi n)^{2} b_{n}^{2}\right\} .
\end{aligned}
$$

The second derivative term in the Liouville action (3.5) disappears in the mode expansion. For the ghost fields, we also take the Neumann boundary condition. We obtain the 
Faddeev-Popov determinant

$$
\left\{\operatorname{det}^{\prime}\left(P_{1}^{\dagger} P_{1}\right)\right\}_{\hat{g}}^{1 / 2}=2 \pi t \prod_{n \neq 0} n \sinh (2 \pi n t) .
$$

There is no zero mode, because it is absent from beginning.

Inserting the partition functions of conformal matter fields and the Liouville field (3.8), (3.12) and the Faddeev-Popov determinant (3.14) into equation (3.1), we obtain finally the transition amplitude for the case of annulus topology explicitly;

$$
\begin{aligned}
Z\left[X_{F}^{\mu}, \phi_{F} ; X_{I}^{\mu}, \phi_{I}\right]= & 2 \pi \int_{0}^{\infty} d t K\left(X_{0 F}^{\mu}, t ; X_{0 I}^{\mu}, 0\right) K\left(\phi_{0 F}, t ; \phi_{0 I}, 0\right) \\
& \times\left\{\prod_{n \neq 0} n \sinh (2 \pi n t)\right\}^{(1-c) / 2} .
\end{aligned}
$$

We can easily show that the partition function for the zero mode and the one for the non-zero modes satisfy the Euclidean Schrödinger equation individually;

$$
\begin{aligned}
& \hat{H}_{0}^{X_{0}^{\mu}} K\left(X_{0 F}^{\mu}, t ; X_{0 I}^{\mu}, 0\right)=-\frac{\partial}{\partial t} K\left(X_{0 F}^{\mu}, t ; X_{0 I}^{\mu}, 0\right), \\
& \hat{H}_{0}^{a_{n}^{\mu}} K\left(a_{n F}^{\mu}, t ; a_{n I}^{\mu}, 0\right)=-\frac{\partial}{\partial t} K\left(a_{n F}^{\mu}, t ; a_{n I}^{\mu}, 0\right)
\end{aligned}
$$

and

$$
\begin{aligned}
& \hat{H}_{0}^{\phi_{0}} K\left(\phi_{0 F}, t ; \phi_{0 I}, 0\right)=-\frac{\partial}{\partial t} K\left(\phi_{0 F}, t ; \phi_{0 I}, 0\right), \\
& \hat{H}_{0}^{b_{n}} K\left(b_{n F}, t ; b_{n I}, 0\right)=-\frac{\partial}{\partial t} K\left(b_{n F}, t ; b_{n I}, 0\right) .
\end{aligned}
$$

The Hamiltonian operators $\hat{H}_{0}^{X_{0}^{\mu}}, \hat{H}_{0}^{a_{n}^{\mu}}, \hat{H}_{0}^{\phi_{0}}$ and $\hat{H}_{0}^{b_{n}}$ are defined as follows. From the mode-expanded actions (3.9) and (3.13), we can rewrite the actions and Hamiltonians;

$$
S_{X^{\mu}}\left[X^{\mu}, t\right]=\int_{0}^{t} d \sigma^{0}\left\{P_{X_{0} \mu} \dot{X}_{0}^{\mu}+\sum_{n \neq 0} P_{a_{n} \mu} \dot{a}_{n}^{\mu}-H_{0}^{X^{\mu}}\right\}
$$

and

$$
S_{\phi}[\phi, t]=\int_{0}^{t} d \sigma^{0}\left\{P_{\phi_{0}} \dot{\phi}_{0}+\sum_{n \neq 0} P_{b_{n}} \dot{b}_{n}-H_{0}^{\phi}\right\}
$$


where

$$
\begin{aligned}
H_{0}^{X^{\mu}} & =\frac{1}{2}\left[P_{X_{0}}{ }^{\mu} P_{X_{0} \mu}+\sum_{n \neq 0}\left\{\dot{a}_{n}^{\mu} \dot{a}_{n \mu}-(2 \pi n)^{2} a_{n}^{\mu} a_{n \mu}\right\}\right] \equiv H_{0}^{X_{0}^{\mu}}+\sum_{n \neq 0} H_{0}^{a_{n}^{\mu}}, \\
H_{0}^{\phi} & =\frac{\kappa}{2}\left[P_{\phi_{0}}^{2}+\sum_{n \neq 0}\left\{\dot{b}_{n}^{2}-(2 \pi n)^{2} b_{n}^{2}\right\}\right] \equiv H_{0}^{\phi_{0}}+\sum_{n \neq 0} H_{0}^{b_{n}} .
\end{aligned}
$$

In order to obtain the Hamiltonian operators, we replace the momentum by the differential operator.

We observe that the last term of the transition amplitude (3.15) becomes a number only when we take $c=1$. This means that there is no vibration of the string as stated in the introduction. Therefore only in the case of $c=1$, we can show that the transition amplitude of the string universe obeys the minisuperspace Wheeler-DeWitt equation, by using the Euclidean Schrödinger equations (3.16) and (3.17);

$$
\begin{aligned}
\hat{H}_{0} Z\left[X_{F}, \phi_{F} ; X_{I}, \phi_{I}\right] & =\left(\hat{H}_{0}^{X}+\hat{H}_{0}^{\phi}\right) Z\left[X_{F}, \phi_{F} ; X_{I}, \phi_{I}\right] \\
& =\left(\hat{H}_{0}^{X_{0}}+\hat{H}_{0}^{\phi_{0}}\right) Z\left[X_{F}, \phi_{F} ; X_{I}, \phi_{I}\right] \\
& =-2 \pi \int_{0}^{\infty} d t \frac{\partial}{\partial t}\left\{K\left(X_{0 F}, t ; X_{0 I}, 0\right) K\left(\phi_{0 F}, t ; \phi_{0 I}, 0\right)\right\} \\
& =-2 \pi\left[K\left(X_{0 F}, t ; X_{0 I}, 0\right) K\left(\phi_{0 F}, t ; \phi_{0 I}, 0\right)\right]_{0}^{\infty} \\
& =\delta\left(X_{0 F}-X_{0 I}\right) \delta\left(\phi_{0 F}-\phi_{0 I}\right) .
\end{aligned}
$$

Here, in the case of $c=1$, the reason why we could ignore the cosmological constant is clear by following the discussions in Ref. [8] [11; the theory cut-off by the exponential interaction which originates from the cosmological constant may be identified with the free field theory with an appropriate renormalization. We do not have to take the modification of the Liouville field dynamics proposed by David and by Distler and Kawai [12, [13], when we only consider the annulus topology without cosmological constant.

As is well known, the quantity $\prod_{n \neq 0} n \sinh (2 \pi n t)$ in the Faddeev-Popov determinant diverges. If we regularize this quantity by using $\zeta$ function, we obtain the well-known 
form

$$
\begin{aligned}
\left\{\operatorname{det}^{\prime}\left(P_{1}^{\dagger} P_{1}\right)\right\}_{\hat{g}}^{1 / 2} & =2 \pi t \prod_{n \neq 0} n \sinh (2 \pi n t) \\
& =2 \pi t e^{-\frac{\pi t}{3}} \prod_{n=1}^{\infty}\left(1-e^{-4 \pi n t}\right)^{2}
\end{aligned}
$$

This regularization is not important, because these divergent quantities are all cancelled out in the transition amplitude (3.15) in the case of $c=1$.

On the other hand, if we take the Dirichlet boundary condition for the conformal matter fields and the Liouville field, we obtain the transition amplitude;

$$
\begin{gathered}
Z\left[X_{F}^{\mu}, \phi_{F} ; X_{I}^{\mu}, \phi_{I}\right] \\
=2 \pi \int_{0}^{\infty} d t K\left(X_{0 F}^{\mu}, t ; X_{0 I}^{\mu}, 0\right) K\left(\phi_{0 F}, t ; \phi_{0 I}, 0\right) \prod_{n \neq 0} n^{(3+c) / 2}\{\sinh (2 \pi n t)\}^{(1-c) / 2} \\
\times \exp \left[-\sum_{n \neq 0} \frac{\pi n}{\sinh (2 \pi n)}\left\{\cosh (2 \pi n t)\left(a_{n F}^{\mu} a_{n F \mu}+a_{n I}^{\mu} a_{n I \mu}+b_{n F}^{\mu} b_{n F \mu}+b_{n I}^{\mu} b_{n I \mu}\right)\right.\right. \\
\left.\left.-\left(2 a_{n F}^{\mu} a_{n I \mu}+2 b_{n F}^{\mu} b_{n I \mu}\right)\right\}\right] .
\end{gathered}
$$

The Dirichlet boundary condition is not suited even for the $c=1$ case. One reason is that if we regularize the quantity $\prod_{n \neq 0} n^{2}$, it becomes zero and the transition amplitude given by equation (3.23) becomes meaningless. The other reason is as follows. In the Dirichlet case, we are left with the non-zero modes of the conformal matter field and those of the Liouville field on the boundary. In addition, the factor $\sinh ^{-1}(2 \pi n)$ is cancelled out by the Faddeev-Popov determinant, and therefore the non-zero modes on the boundary cannot satisfy the Euclidean Schrödinger equations (3.16), (3.17) by using the parts which do not cancel out. In this case, the transition amplitude (3.23) cannot obey the Wheeler-DeWitt equation. These are the reasons why we have chosen the Neumann boundary condition.

Our result depends crucially on the fact that we have considered the case with the $c=1$ conformal matter field. The situation for $c \neq 1$ is beyond the scope of this paper, because it is impossible to cancel out the vibrations of the non-zero modes. 


\section{Conclusion}

In this paper, we have pointed out that there is lack of canonical structure in twodimensional gravity [7]. In order to eliminate the problematic term, we have taken a conformal gauge by concentrating on the case of annulus topology. Our main result is the transition amplitude of the string universe (3.15) which obeys the minisuperspace Wheeler-DeWitt equation (3.21). We have restricted ourselves to the case of the $c=1$ conformal matter field and have imposed the Neumann boundary condition.

There may be other methods to avoid this problem, and it is worth investigating different gauge fixing (for example Ref.[14]).

Acknowledgments

We would like to express our gratitude to Prof. Itoyama and Prof. Kubota for their helpful discussions. Thanks are also due to Prof. Itoyama for a careful reading of the manuscript and the other members of High Energy Physics group in Osaka University for their warm encouragements.

\section{References}

[1] J.J. Halliwell, lecture delivered at the Jerusalem Winter School on Quantum Cosmology and Baby Universes (1990).

[2] J.J. Halliwell, Phys. Rev. D38 (1988) 2468

[3] E. D’Hoker and D.H. Phong, Rev. Mod. Phys. 60 (1988) 917

[4] G. Moore, N. Seiberg and M. Staudacher, Nucl. Phys. B362 (1991) 665

[5] N. Seiberg, Prog. Theor. Phys. Suppl. 102 (1990) 319

[6] C. Teitelboim, Phys. Lett. B126 (1983) 41 
[7] A. Ishikawa, H. Itoyama and T. Kubota, unpublished

[8] M. Bershadsky and I.R. Klebanov, Phys. Rev. Lett. 65 (1990) 3088

[9] T. Yoneya, Prog. Theor. Phys. Suppl. 85 (1985) 256

[10] E. Martinec, Phys. Rev. D30 (1984) 1198

[11] I.R. Klebanov, lectures given at Spring School on String Theory and Quantum Gravity, Trieste, Italy (1991).

[12] F. David, Mod. Phys. Lett. A3 (1988) 1651

[13] J. Distler and H. Kawai, Nucl. Phys. B321 (1989) 509

[14] D.Z. Freedman, J.I. Latorre and K. Pilch, Nucl. Phys. B306 (1988) 77 